C. WATER VAPOR ABSORPTION 


\section{THE SPECTROSCOPIC SEARCH FOR WATER ON MARS:}

\section{A HISTORY}

RONALD A. SCHORN

Jet Propulsion Laboratory, California Institute of Technology, Pasadena, Calif., U.S.A.

The discovery of water on Mars is a lot like the discovery of America. Both were done many times by many different people. In both cases many of the 'discoveries' were either fictitious or difficult to verify. Regarding the discovery of America, a grammar school teacher of mine once pointed out that Columbus was the effective discoverer of America. After he did it, people believed it was so. More importantly, people began to act on the basis of his discovery.

Curiously, the effective discovery of water on Mars did not take place until 1963. I say curiously, because for centuries, it seemed that definitive proof was 'just around the corner'. The history of this search for evidence is most interesting in itself. As a good deal of the observational work and speculation on the subject was wrong, it has the additional quality of being a tale with a moral (for astronomers and many others). Finally, it has a happy ending.

One hundred years ago, it seemed obvious that there was a great deal of water on Mars. There was plenty of water on Earth, and telescopic observations showed that Mars resembled the Earth. Mars had polar caps that varied in size with the seasons, clouds that came and went, continents (the bright or 'red' areas), and great bodies of water (the dark areas). While the Beer Sea and the Dawes Ocean are long gone, Mare Sirenum, Mare Tyrrhenum, and the like are more permanent reminders of this state of our knowledge.

Figure 1 reproduces a plate from Other Worlds Than Ours by the well known 19th-century popularizer of astronomy, Richard A. Proctor (1873). The chart was made from the observations of Dawes, and many permanent features of Mars are recognizable. For example, the Kaiser Sea is Syrtis Major, J. Herschel Strait is Sinus Sabaeus, etc. The interpretation of these features, however, has changed completely since the 1870's. Even in those days, however, there were those who, remembering the fate of the lunar 'seas', weren't too sure that the Martian seas were full of water. Of course, the existence of a Martian atmosphere and polar caps made the existence of water on Mars more probable than in the case of the Moon, but more direct evidence was needed. So, beginning in the 1860's, "The wonderful powers of the spectroscope have been applied to this question," to quote Proctor.

The first observations were made, of necessity, with the visual spectroscope. In 1867 Huggins, by comparing the spectra of Mars and the Moon in the orange region, became the first 'discoverer' of water vapor in the atmosphere of Mars. Thus began a dreary series of observations which were all wrong, misleading, indecisive, or negative. The important point about all of the late 19th- and early 20th-century observations was that the upper limit of the abundance of Martian atmospheric water vapor was

16-P.A.

Sagan et al. (eds.), Planetary Atmospheres, 223-236.

All Rights Reserted. Copiright 197 I by the I.A.l 

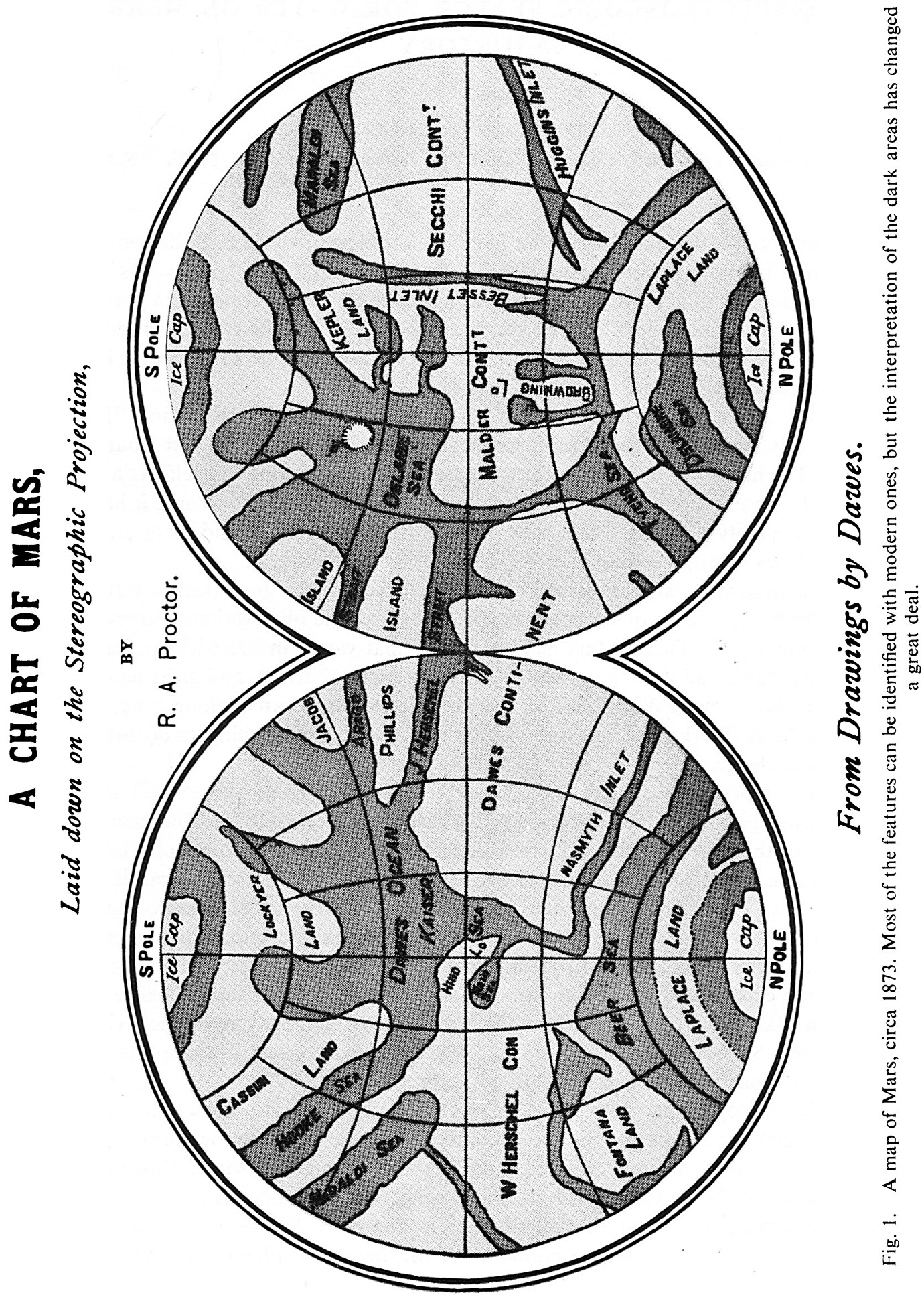
pushed steadily downward. The course of this story through the early years is given by Campbell (1909) and carried up to the middle of the twentieth century clearly and concisely by de Vaucouleurs (1954) in his landmark book on Mars, so will not give all the details here.

The photographic plate replaced the human eye as the recorder of spectra during this period, and observations were made at higher and dryer sites as time went on. Also, the comparison of 'band strengths' on low dispersion spectra gave way to highdispersion spectra. On the latter, the Doppler shift (due to the relative radial velocity of Mars and the Earth) could be used to separate any Martian water lines from their terrestrial counterparts or, at least, to cause an asymmetry in the shape of the terrestrial water-vapor lines.

The Doppler-shift technique was first attempted by Lowell and Slipher (1905), and later by Campbell and Albrecht (1910), in the orange and red regions of the spectrum. Whether or not Martian water vapor was detected, exactly how much was, and what the upper limits were for these early studies, has never been settled. However, it is probably fair to say that all of these observations agreed that the amount of Martian water vapor per unit area (if it existed at all) was small in comparison to the amount in the Earth's atmosphere.

Meanwhile, the idea of Martian seas had been dying out as observational evidence against this concept accumulated. Russell et al. (1945) summarize this evidence. First, the brilliant reflection of the Sun from an open-water surface has never been seen. Second, the dark regions exhibit a great deal of internal structure. Finally, the dark areas show variation in shade and depth of color, and size and shape, during the march of the Martian seasons, and from year to year. This left the pole caps and various transient white 'clouds', 'frosts', and 'mists' as indirect evidence for water on Mars.

Considering the rapid shrinking in estimates of the amount of Martian water it is not surprising that, at the turn of the century, Stoney (1898) suggested frozen carbon dioxide for the composition of the polar caps and various white patches on Mars. At the time, and for long afterwards, there was no direct evidence that carbon dioxide (in large amounts) existed on Mars. But, during the next seventy years, the suggestion had varying degrees of popularity, and was never disproved.

There was a lack of definite spectroscopic evidence for the existence of any gas in the atmosphere of Mars (and in particular, water vapor) during the first half of the twentieth century. In this interval, of necessity, a great deal of work on possible and probable abundances was carried out based on 'less-direct' techniques. Some examples are photometric and polarimetric observations, calculations based on the existence and some assumed composition of the polar caps and clouds, and the like. Only a few examples of this large amount of work (large compared to the amount of spectrographic evidence) will be covered in the course of this review. First, there is not the time to do so here. Second, they are discussed in detail in de Vaucouleurs' book. Finally, I must say, most of these papers were not of much help in solving the problem of obtaining definite evidence for the existence of water on Mars. Usually they predicted amounts just below whatever the current spectroscopic limit was at the time.

The 'modern era' in spectroscopic observations of Mars began in 1925 when 
Adams and St. John (1926) used the îrst coudé spectrograph - a six-prism instrument on the Mt. Wilson 60 -inch reflector. Their resultant dispersion was $7.3 \AA / \mathrm{mm}$ near $6300 \AA$. They attempted to measure the displacement in wavelength of strong terrestrial $\mathrm{H}_{2} \mathrm{O}$ lines which would be caused by any (presumably unresolved) weaker Martian $\mathrm{H}_{2} \mathrm{O}$ components. Such a displacement was detected and, in present day terms, corresponded to about $450 \mu$ of precipitable $\mathrm{H}_{2} \mathrm{O}$ over unit area on Mars. This result was almost certainly wrong. For one thing, their measures of the $\mathrm{O}_{2}$ band near $6300 \AA$ showed Martian components one-third the strength of the terrestrial $\mathrm{O}_{2}$ lines, which was certainly an error. Secondly, the $\mathrm{H}_{2} \mathrm{O}$ lines available in the region of the spectrum they used are quite weak compared to those further out in the infrared (e.g. $8200 \AA$ ). The amount of water on Mars in the 1960 's would have produced Martian components far below the detectability limit of the 1925 spectra. While it is possible that the amount of Martian atmospheric water vapor has decreased an order of magnitude in $40 \mathrm{yr}$, it is most unlikely.

The availability of speculum-metal diffraction gratings made possible a more ambitious test in 1933 (Dunham, 1952), which gave negative results for lines "just beyond the tail of the B band of oxygen." Finally, the 'modern' coudé spectrograph emerged in 1936, when Wood made available gratings ruled on aluminized Pyrex disks. Using one of these gratings at the coudé focus of the 100-inch reflector, Adams and Dunham obtained a number of spectra of Mars in the region of the $8200 \AA \mathrm{H}_{2} \mathrm{O}$ band. The results were uniformly negative, and have been published in a number of places (Adams and Dunham, 1937; Adams, 1941; Dunham, 1952). This work was neatly summarized by Dunham in his 1952 paper. There he set a probable upper limit of about $10 \mu$ of precipitable water vapor in the atmosphere of Mars. This upper limit was probably too low. Hess $(1951,1961)$ points out that the true limit from these old Mt. Wilson plates is probably much higher, somewhere between 10-100 $\mu$. First of all, the measurement of line asymmetries was not made. Secondly, the curve of growth for $\mathrm{H}_{2} \mathrm{O}$ was not employed (that for $\mathrm{O}_{2}$ was used) and thirdly, the dependence of $\mathrm{H}_{2} \mathrm{O}$ absorptions on pressure was not considered. Finally, Schorn et al. (1969) have shown that the old Mt. Wilson plates used by Adams and Dunham in the near-infrared are comparable to modern I-N emulsions in grain size and resolution. In the $8200 \mathrm{H}_{2} \mathrm{O}$ bands the strongest Martian $\mathrm{H}_{2} \mathrm{O}$ lines so far observed have equivalent widths of about $5 \mathrm{~m} \AA$. At the dispersion used by the Mt. Wilson workers $(6 \AA / \mathrm{mm} @ 7200 \AA)$ these lines would be invisible on I-N emulsions, a rough detection limit being $10 \mathrm{~m} \AA$. Thus the old Mt. Wilson upper limits were doubtlessly too strict, 50 to $100 \mu$ being more realistic. (In 1954, de Vaucouleurs suggested $\ll 350 \mu$, possibly $100 \mu$.) With this modest effort, high-dispersion spectroscopic work in this area ended for $20 \mathrm{yr}$.

In 1947-48 Kuiper $(1947,1952)$ was the first to record the spectrum of Mars between 1.0 and $2.5 \mu$, with a low-resolution (numerical resolution of 80 ) infrared spectrometer. Two $\mathrm{CO}_{2}$ absorption bands near $1.6 \mu$, and three $\mathrm{CO}_{2}$ bands near $2 \mu$, were considerably enhanced in the Martian spectrum when compared to Lunar spectra taken under similar circumstances. This indicated that the Martian $\mathrm{CO}_{2}$ abundance was comparable to that of Earth, firmly establishing $\mathrm{CO}_{2}$ as the first proven component of the Martian atmosphere. These are 'strong' $\mathrm{CO}_{2}$ bands and their intensities (line 
profiles were not resolved in this pioneering work) depend on the abundance of $\mathrm{CO}_{2}$ and the effective pressure. At the time, a $\mathrm{CO}_{2}$ abundance about twice that of the Earth's atmosphere (twice $220 \mathrm{~cm}$-atm or about $440 \mathrm{~cm}$-atm), and a Martian surface pressure of a little less than $1 / 10 \mathrm{~atm}$ were reasonably consistent with Kuiper's data and our knowledge of Mars. This estimated $\mathrm{CO}_{2}$ abundance led to a partial pressure of $\mathrm{CO}_{2}$ at the surface of Mars of about $\frac{1}{4} \mathrm{~mm} \mathrm{Hg}$. This in turn led to a $\mathrm{CO}_{2}$ equilibrium temperature of $129 \mathrm{~K}$, much lower than any estimates of the cap temperature. At this time then, it seemed that dry-ice polar caps were ruled out, and water-ice caps strongly indicated. Of course, we now know that the $\mathrm{CO}_{2}$ abundance is much higher than Kuiper's early estimate, and the surface pressure on Mars much lower than de Vaucouleurs $85 \pm 4 \mathrm{mb}$ estimate. The new determinations are consistent with Kuiper's original data and our present knowledge of Mars, and actually suggest $\mathrm{CO}_{2}$ caps now (Leighton and Murray, 1968) - but this is a subject for a different review paper. Nevertheless, we have here a good example of how shaky 'solid' evidence can be especially when it comes to interpretation.

As the Martian $\mathrm{H}_{2} \mathrm{O}$ abundance was known to be much less than that of Earth, low-resolution spectroscopy from the Earth's surface was not expected to show any evidence of Martian $\mathrm{H}_{2} \mathrm{O}$ absorption. However, Kuiper also obtained reflection spectra (at a low resolution of 9) of the polar caps themselves - a most difficult set of observations. The Martian cap spectra resembled the transmission spectrum of a water cell of $1 \mathrm{~mm}$ thickness. Kuiper noted that (thin) $\mathrm{H}_{2} \mathrm{O}$ frost deposits on dry ice $\left(-78^{\circ} \mathrm{C}\right.$, $1 \mathrm{~atm}$ ) also showed a water cell equivalent of $1 \mathrm{~mm} .{ }^{*}$ He concluded that the polar caps were frozen $\mathrm{H}_{2} \mathrm{O}$ at temperatures much below $0{ }^{\circ} \mathrm{C}$. Later, Moroz (1964) confirmed these observations. But, as Leighton and Murray (1968) pointed out, we do not know enough about the reflection spectrum of both solid $\mathrm{H}_{2} \mathrm{O}$ and $\mathrm{CO}_{2}$, under Martian conditions, to draw a definite conclusion.

At the same time Hess $(1948,1951,1961)$ tackled the problem of whether the low spectroscopic $\mathrm{H}_{2} \mathrm{O}$ abundance estimates would allow the production of the clouds seen on Mars. Considering low level clouds (morning limb haze, etc.), and assuming the water to be concentrated in a layer $\frac{1}{2} \mathrm{~km}$ deep near the ground (not at all impossible, in the light of 1969 observations) he derived an upper limit of $400 \mu$ of precipitable $\mathrm{H}_{2} \mathrm{O}$. A treatment of possible high-level convective (afternoon) clouds led to an upper limit of $600 \mu$. He pointed out that the mean $\mathrm{H}_{2} \mathrm{O}$ content would be considerably less, as such clouds are indeed rare. De Vaucouleurs, after discussing Hess' work, makes an order-of-magnitude estimate of the Martian atmospheric $\mathrm{H}_{2} \mathrm{O}$ abundance based on the assumption that the polar caps are thin layers of water ice. His result was $<100 \mu$, quite consistent with the best spectroscopic results at the time. De Vaucouleurs quite candidly stresses the large uncertainties of his and other estimates, and cautions against taking agreement too literally.

In 1956 Kiess et al. (1957), in a most ingenious manner, obtained high-dispersion spectra of Mars without using a large telescope. They utilized a large grating spectrograph fed by a 12-inch uranostat. The lack of a large telescope enabled them to set up their equipment near the summit of Mauna Loa, Hawaii - the highest, driest site yet

* In the light of our present knowledge (1970) this statement is remarkably prophetic. 
used for such work. Unfortunately, the resultant image size was quite small $-0.3 \mathrm{~mm}$. This, together with the use of relatively grainy 103aF, (ammoniated) 103-U, and (ammoniated) $\mathrm{I}-\mathrm{N}$ plates resulted in narrow spectra with a low signal to noise ratio. Thus, even though the terrestrial water lines were quite weak, the (as we now know) very weak Martian lines would still be invisible. The ' $a$ ' band of $\mathrm{H}_{2} \mathrm{O}$ was observed at $2 \AA / \mathrm{mm}$ from Mauna Loa, but the stronger 8200 band was observed only at $5 \AA / \mathrm{mm}$ from Georgetown, Maryland, a much wetter location. By comparing their observations with laboratory spectra, Kiess et al. set an upper limit of $80 \mu$ for the Martian $\mathrm{H}_{2} \mathrm{O}$ abundance.

A number of less direct studies of water on Mars should now be mentioned. Observations by Lyot (1929) and Dollfus (1948, 1951, 1961) have shown that the polarimetric properties of a polar cap are exactly similar to that of finely granulated $\mathrm{H}_{2} \mathrm{O}$ frost deposited in a cold (liquid air), low pressure $(6 \mathrm{~cm} \mathrm{Hg}$ ) environment. Further, Dollfus found that the polarization of bright patches at the edge of a polar cap is closely matched by terrestrial ice clouds. However, Leighton and Murray later noted that, as Dollfus had not studied the polarization properties of $\mathrm{CO}_{2}$ frost, his conclusions were far from definite.

Urey (1959), from the existence of (assumed $\mathrm{H}_{2} \mathrm{O}$ ) early-morning haze, suggested a lower limit to the $\mathrm{H}_{2} \mathrm{O}$ atmospheric abundance of about $40 \mu$. From the sublimation rate of a (supposed water ice) polar cap, and the Martian greenhouse effect Sagan (1961) suggested values of 20-200 $\mu$ for the Martian water-vapor abundance. Lebedinskii and Salova (1962), considering the evaporation rate of the pole caps and the degree of atmospheric transparency on Mars, arrived at a mean $\mathrm{H}_{2} \mathrm{O}$ abundance of $14 \mu$.

In 1962 Adamcik (1963) presented calculations "of the expected water vapor content of the Martian atmosphere on the assumption that it is determined by the equilibrium dissociation pressure of ferric oxide hydrate." Lower and upper limits of about $0.3-60 \mu$ were obtained. These last few results are startlingly close to modern observational results, but the coincidence may be just that. When we know more about Mars we will be able to judge.

In this connection we must mention the interesting suggestion of Kiess et al. (1960, 1962, 1963). Based on their inability to detect any Martian $\mathrm{H}_{2} \mathrm{O}$, and their belief that absorption lines due to the oxides of nitrogen were visible on their plates (Kiess et al., 1962) they proposed that the latter were responsible for a wide variety of phenomena on Mars - including many of those used to 'prove' the presence of water (polar caps, clouds, etc.). Unfortunately, further spectroscopic tests (e.g. Sinton, 1961 ; Spinrad, 1963; Marshall, 1964) failed to detect any trace of the oxides of nitrogen. In particular Spinrad, working in the photographic region of the spectrum, as did Kiess et al., but using wider spectra and finer-grain plates, set an upper limit of $1 \mathrm{~mm}$ atm. Finally, Sagan et al. derived an upper limit of $1 \mathrm{~mm}$ atm for the $\mathrm{NO}_{2}$ abundance by computing the photochemical equilibria of nitrogen oxides on Mars under the action of solar UV and visible light. They point out that the presence of water (which is now proven) would lower this limit further. It seems probable that Kiess and his collaborators were misled by their narrow, grainy spectra and did not, in fact, see any absorption features due to the oxides of nitrogen. 
In 1962 Spinrad and Richardson (1963) analyzed an excellent coudé spectrum of Mars obtained with the Victoria 48-inch reflector. The terrestrial $\mathrm{H}_{2} \mathrm{O}$ abundance was very much lower than usual at this usually sodden site. Unfortunately, the relatively weak $7200 \AA \mathrm{H}_{2} \mathrm{O}$ band (weak compared to the $8200 \AA \mathrm{H}_{2} \mathrm{O}$ band) and a low resolution (low compared to IV-N) I-N emulsion were used, and the Doppler shift was only $-0.29 \AA$. As a result, all the authors could state was that there were no Martian $\mathrm{H}_{2} \mathrm{O}$ components with strengths $>9 \mathrm{~m} \AA$. We now know that lines $>9 \mathrm{~m} \AA$ in this band would not be expected.

At this point, in 1963, the full weight of modern, high-dispersion spectroscopy was, for the first time, turned on this problem. Between World War I and 1963 planets had been the stepchildren of astronomy - they definitely were not in the 'mainstream'. There were many reasons. First of all, extra-solar-system astronomy 'exploded': the structure of the Milky Way Galaxy was roughed out, the existence of external galaxies was demonstrated, the red-shift of galaxies was discovered, and stellar evolution studies began to show substantial results. After millennia of dealing almost entirely with the solar system, astronomers' attention was directed elsewhere. Secondly, planetary astronomy had hit an apparent dead end. New results were hard to come by (this review illustrates a particular case) and telescopes were few - too few to 'waste' much time on such a small portion of the universe. Finally, there was an element of irrational prejudice against planetary astronomy - no doubt generated by Lowell's Martians, Flammarion's poisonous comet tales, and Orson Wells' radio coup. Astronomers used to have a shy and retiring image, and 'life on Mars' may have been too much for them.

Regardless of the reasons, very little 'big-telescope' time was devoted to the planets during the (say) 1915-1965 period. Adams and Dunham, for example, note that most of their planetary spectra were taken when the seeing was too poor for stellar spectra! The catalyst which changed this situation was provided by Spinrad. His work on the old Mt. Wilson and Victoria plates convinced him that very fine-grained IV-N emulsions, used with spectrographs of the quality of the Mt. Wilson 100-inch coudé, would provide substantial new results. In addition, he had found that the IV-N emulsion, usually considered much too slow for useful astronomical work, could be ammonia-sensitized to a speed equal to that of the I-N emulsion.

While Spinrad was trying to get some coudé time Dollfus (1963) used conventional astronomical photometry of the strong $1.4 \mu \mathrm{H}_{2} \mathrm{O}$ band at a high and dry mountain site. The location compensated for low spectral resolution of the photoelectric spectrophotometer and birefringent filter system. This was essentially a return to turn of the century techniques, with the important differences of a much dryer site and the use of a much stronger $\mathrm{H}_{2} \mathrm{O}$ band. Dollfus' observations were made in January of 1963 when $L_{s}{ }^{*}$ was about $40^{\circ}$. The initial result (Dollfus, 1963) gave an average, over the visible disk of Mars, of $200 \mu$ of precipitable water vapor. This result seemed much too large at the time, as it was above the Adams and Dunham abundance limit and

* $L_{s}$ is the planetocentric longitude of the Sun. $L_{s}=0$ at the vernal equinox, or beginning of spring in the northern hemisphere of Mars; $L_{s}=90^{\circ}$ at the beginning of summer in the northern hemisphere of Mars, etc. 
also above that of Spinrad and Richardson set just the month before. It is important, in this regard, to note that detection and abundance estimates are two different things. Later, Dollfus $(1964,1965)$, using better laboratory data and newer, more accurate values of the Martian surface pressure, obtained values of $150 \mu$ and, ultimately, $45 \mu$. This last result, as we shall see, still seems too high by about a factor of two, especially for the value of $L_{s}$ involved, and this might illustrate an inherent limit to the accuracy of this method. And yet, in the light of our current knowledge of Mars, and the absence of any conflicting data from January, 1963, the result may be correct. It is however unfortunate that the first detection announcement was widely disbelieved because the abundance estimate was so high.

On April 12/13, $1963\left(L_{s}=76^{\circ}\right)$ Spinrad et al. (1963), Kaplan et al. (1964) obtained a good IV-N plate of the $8200 \AA \mathrm{H}_{2} \mathrm{O}$ band at $5.6 \AA / \mathrm{mm}$ using the 100 -inch coudé spectrograph. The Doppler shift was $+0.414 \AA$ (at $8200 \AA$ ) at that time, and a number of 3-5 mA Martian $\mathrm{H}_{2} \mathrm{O}$ lines were seen. A first estimate of 5-10 $\mu$ of $\mathrm{H}_{2} \mathrm{O}$ on Mars was made by guessing at the line strengths of the $\mathrm{H}_{2} \mathrm{O}$ lines involved and also by comparing the Martian and terrestrial lines, using the known amount of (terrestrial) water above Mt. Wilson that night, and assuming a curve of growth. Shortly thereafter Rank et al. (1964) measured the intrinsic strengths of a number of the lines in the $8200 \AA$ band. Using these line strengths, the Mt. Wilson plate gave $14 \pm 7 \mu$ as a final result.

On this same plate the weak $8700 \AA$ band of $\mathrm{CO}_{2}$ was detected, showing at once that the Martian $\mathrm{CO}_{2}$ abundance was much higher than previously thought. More importantly, the surface pressure was drastically reduced. In the Space Age this result had great practical importance, and the furor it generated led to a great deal of importance being laid on the single KMS plate. The $\mathrm{KMS} \mathrm{H}_{2} \mathrm{O}$ result shared in this attention, and can be considered the effective discovery of water on Mars. We shall see, however, that not everyone was convinced. Heyden et al. (1966) called particular attention to the possibility of weak telluric or solar lines confusing the issue. Clearly, more observations were needed.

During the 1964-65 apparition of Mars Schorn et al. (1967) carried out a program on the $8200 \AA \mathrm{H}_{2} \mathrm{O}$ band using the 120-inch Lick and (newly improved) 82-inch McDonald Observatory coudé spectrographs. Using IV-N emulsions and dispersions of about $4 \AA / \mathrm{mm}$, nineteen excellent spectra were obtained. The presence of Martian $\mathrm{H}_{2} \mathrm{O}$ was confirmed, and variations with the season on Mars, and over the disk of the planet, were noted. Using Rank's values for line intensities the results were: (a) between $L_{s}=5^{\circ}$ and $L_{s}=31^{\circ}$ (i.e. early northern spring), $<15 \mu$ everywhere; (b) between $L_{s}=47^{\circ}$ and $L_{s}=61^{\circ}$, (i.e. late northern spring), $\sim 15 \mu$ in the northern hemisphere only (i.e. over the receding polar cap); between $L_{s}=110^{\circ}$ and $L_{s}=122^{\circ}$ (i.e. northern summer, southern winter), $\sim 10 \mu$ everywhere, with one plate at $L_{s}=122^{\circ}$ giving $\sim 25 \mu$ in the southern hemisphere. These results seemed to show that some of the pole cap, at least, was composed of frozen $\mathrm{H}_{2} \mathrm{O}$ while the very presence of $\mathrm{H}_{2} \mathrm{O}$ vapor implied an atmospheric temperature greater than $200 \mathrm{~K}$ in the absorbing layer.

We felt that the spectroscopic study of Martian $\mathrm{H}_{2} \mathrm{O}$ was not finished by these results, but just begun. Higher spectral and areographic resolution, we felt, would give 
most interesting results (one superb spectrum obtained on December 24, 1964, showed $\mathrm{H}_{2} \mathrm{O}$ only above the North polar cap and Mare Acidalium!). Increasing the range of $L_{s}$ observed, and comparing the Martian weather from year to year were clearly desirable. Finally, I must admit that the evidence was far from overwhelming, and many remained unconvinced. For example, Goody (1969) referred to the 'debatable claim' of $15 \mu$ of Martian $\mathrm{H}_{2} \mathrm{O}$. Further, variations over the planet might just be explained away as due to the varying albedo of Mars, the characteristic curve of the IV-N emulsion, etc., while supposed variations of the $\mathrm{H}_{2} \mathrm{O}$ abundance estimates with $L_{s}$ might be due to the varying terrestrial humidity, wishful thinking on the part of the estimators, etc. There was certainly room for improvement in the observations.

During the 1966-67 apparition Schorn and Moore and Owen used the McDonald 82-inch in an attempt to extend the 1964-65 work. Unfortunately, snow, rain, clouds, and high terrestrial humidity ruined the program. For the 1969 apparition, the outlook was considerably better. A larger collimator made a dispersion of $2 \AA / \mathrm{mm}$ attainable with the 82-inch coudé spectrograph.

At about this time Leighton and Murray (1966) and Leovy (1966) revived the $\mathrm{CO}_{2}$-pole-cap hypothesis with some force, basing their work on new data for the $\mathrm{CO}_{2}$ abundance and surface pressure on Mars. While this work threw no direct light on the validity of the recent $\mathrm{H}_{2} \mathrm{O}$ detections, it certainly made them seem more doubtful in the eyes of many.

In 1967 Kuiper began using a series of jet-plane flights at high altitudes (around $40000 \mathrm{ft}$ ), to perform astronomical observations in the infrared. This logical continuation of the high-altitude observatory idea is still continuing. Using interferometric techniques, he is observing a number of astronomical objects, among them Mars. While the observations have not yet been published, they show definite evidence for the presence of Martian water vapor in the $1.3 \mu$ band. While Kuiper's observations could not give the areographic resolution attainable with large telescopes on the ground, they provided strong support for the feeling that continued study of Martian $\mathrm{H}_{2} \mathrm{O}$ would indeed be useful.

Starting in late 1968 an intensive observational attack on the Martian $8200 \AA \mathrm{H}_{2} \mathrm{O}$ band was carried out at McDonald Observatory. The 82-inch coudé spectrograph was used intensively and, when it became operational, the 107-inch coudé. Some of these results have been discussed in this symposium, other have been published, and the rest will shortly be in print.

In late November and early December of $1968\left(L_{s} \sim 80^{\circ}\right)$ I obtained the first usable spectra of this apparition at the 82-inch. Although the apparent size of Mars was small ( $\sim 9$ arc sec), the seeing poor, and the signal-to-noise ratio of the spectra low, Martian components were clearly seen. These early spectra were taken at $2 \AA / \mathrm{mm}$ and at $4 \AA / \mathrm{mm}$ (these latter duplicating the 1964-65 work), both dispersions showing the strongest Martian lines to be about $5 \mathrm{~m} \AA$, stronger than in 1964-65.

During February, March, and April of $1969\left(L_{s}=110-150^{\circ}\right)$ Little and Schorn, Owen, and Tull obtained a large number of spectra on the 82- and 107-inch telescopes. All were aided by superb, dry weather, a good Doppler shift (almost $+0.5 \AA$ at best), and excellent condition of the spectrographs. Owen and Mason (1969) obtained 
several spectra near $L_{s}=120^{\circ}$. The seeing was poor, so their results referred to a wide, 'pole-to-pole' band on Mars. Using Rank's data for line strengths, Owen obtained an abundance of $35 \pm 15 \mu$. He attributed most of the apparent increase in Martian $\mathrm{H}_{2} \mathrm{O}$ abundance over the 1964-65 results to an increase in the line equivalent widths caused by the greater spectral resolution of the later results. This is of course a very important point. However, the December, 1968 spectra (taken at $2 \AA / \mathrm{mm}$ and $4 \AA / \mathrm{mm}$ ) showed no such systematic effect. As a check, several spectra of the weak lines in the $7820 \AA$ $\mathrm{CO}_{2}$ band of Venus were taken (as close together in time as possible!) later in 1969, at both dispersions. When reduced in the same way as the Martian $8200 \AA$ spectra, there was no systematic difference in the derived equivalent widths between the two dispersions (Schorn and Gray, 1970). I feel that there probably is more water in the Martian atmosphere in 1969 than there was in 1964-65.

Other spectra made in this interval enjoyed better seeing, and the non-uniformity in the Martian humidity was striking. Schorn et al. (1970), in a preliminary step, analyzed spectra taken at $L_{s}=111^{\circ}$ and $122^{\circ}$. As there were many more Martian $\mathrm{H}_{2} \mathrm{O}$ lines visible in these plates than had been the case with earlier observations, it was imperative that additional lines (other than the four Rank's group had done) be observed in the laboratory. Farmer (1970) did this. Further, he corrected the laboratory strengths for temperature by using the lower state energies from Benedict's (1969) assignments for the atlas of Delbouille and Roland (1963). A surprisingly large correction was discovered. For example, the $8197.704 \mathrm{H}_{2} \mathrm{O}$ line increases in strength by a factor of 1.7 between room temperature and $200 \mathrm{~K}$. The result is to lower all $\mathrm{H}_{2} \mathrm{O}$ abundances based on Rank's laboratory data. The KMS result is reduced to about $10 \mu$, and the 1964-65 values are also reduced to about two-thirds of their published values. Owen's result is pushed down toward $30 \mu$. The two 1969 plates at $L_{s}=111$ and $122^{\circ}$ gave $26 \pm 5 \mu$ in the northern (cap-receding) hemisphere, and less than onethird of that amount in the south. The visibility of so many lines enabled an estimate of $225 \mathrm{~K}$ to be made for the effective temperature. This is significantly higher than the $200 \mathrm{~K}$ temperature estimated by Young (1969) for the bulk of the Martian $\mathrm{CO}_{2}$, and suggests that the water vapor exists in a layer close to the ground.

The results described by Little in this Symposium fill in the picture during the February-April period. The preponderance of water in the north was quite marked, and there was little apparent change in the situation over this interval. Also in this Symposium, and elsewhere (Tull, 1970), had discussed his 107-inch plates $\left(L_{s}=\right.$ $\left.132,148^{\circ}\right)$. The greater areographic resolution, as some suspected, led to greater differences in observed $\mathrm{H}_{2} \mathrm{O}$ abundances over the planet. It is important to note that all the observers involved feel that still greater abundance variations will be observed with better areographic resolution. All of these 1968-early 1969 studies, however, give a consistent picture of the situation on Mars at that time.

From May through July a hiatus in the $\mathrm{H}_{2} \mathrm{O}$ observations was caused by the low Doppler shift of Mars. Starting with the Mariner VI and VII flybys, spectra were again obtained with the 82 and 107-inch coudé spectrographs by Barker, Woszczyk, Tull, and Schorn. In August and September $\left(L_{s}=200-235^{\circ}\right)$ no Martian water was observed. The terrestrial humidity was high, and the Doppler shift low (about $\frac{1}{4} \AA$ ), so the 
abundance lower limit was relatively high. Nonetheless, it appeared that the mean Martian water vapor abundance was down by at least a factor of two, during this interval, from its preopposition value. In late December of $1969\left(L_{s} \sim 290^{\circ}\right)$ when the south polar cap was receding, $5 \mathrm{~m} \AA$ Martian lines once again appeared, indicating that the water vapor abundance was back to its early 1969 value. Although the Doppler shift had risen to about $\frac{1}{3} \AA$ by mid-December, this slight increase does not appear to be the sole reason for the reappearance of the Martian $\mathrm{H}_{2} \mathrm{O}$ lines. Probably the Martian water vapor did go away and then return. If so, it appeared later in the season than it did when the northern cap was receding in late 1968-early 1969 . While this hemisphere-to-hemisphere difference in the seasonal behavior of $\mathrm{H}_{2} \mathrm{O}$ on Mars needs much more observation to be firmly established, it would not be unexpected. The orbit of Mars is such that the properties of northern and southern hemispheric seasons are notably different - a difference which could be expected to manifest itself in the behavior of the Martian water-vapor abundance.

Observations with the 82-inch and 107-inch into March, 1970 (photographic and photoelectric scanner), as $L_{s}$ passed $340^{\circ}$, show that the situation on Mars is similar to December 1969 (Barker et al., 1970). During this time the angular diameter of Mars was so small ( $\sim 5$ arc sec) that no effective areographic resolution is possible. The mean $\mathrm{H}_{2} \mathrm{O}$ abundance is about $45 \mu$ during this interval, a high value indeed!

In Figure 2, I have attempted to summarize the spectroscopic observations from 1963 through early 1970 . No attempt has been made to indicate where on the planet various specific measures refer to. Rather the figure illustrates that a cyclical behavior of the Martian water vapor appears to be emerging from the observations.* The water vapor seems to appear when a pole cap has receded appreciably from its maximum extent, and disappears when a cap is at that maximum extent. If this correlation holds up under further observation** we will know that Martian water is cycled through the pole caps, in spite of the Mariner VI and VII evidence (Neugebauer et al., 1969) that those caps are predominantly $\mathrm{CO}_{2}$. The Martian polar caps may indeed be vast deposits of frozen seltzer water!

The situation at present can be summed up as follows. Water exists on Mars. The water vapor varies with location on the planet, the season on Mars, and from year to year. The water appears to cycle through the polar caps, which are partly $\mathrm{H}_{2} \mathrm{O}$. The total amount of water in the atmosphere of Mars is at most a few cubic kilometers. Of course this does not include water which may be trapped as permafrost (as suggested by, among others, Leighton and Murray) or deposited in the permanent remnant of the north polar cap.

Does liquid $\mathrm{H}_{2} \mathrm{O}$ exist on Mars? This is properly a topic for another review paper. But, though improbable (Owen, 1969), it is not impossible (Sagan et al., 1968; Schorn et al., 1970).

Sagan, et al. (1968) discussed the situation on the basis of modern water-vapor abundance estimates. In particular, they pointed out that, while open water surfaces

* The point due to Dollfus is troublesome. Perhaps, as was suggested earlier in this paper, it should be lowered further. At any rate, further observations should decide the matter.

** Further observations are needed. 
(lakes, ponds, etc.) were improbable, the case for liquid water occurring in the interstices of loose soil (dirt, gravel, sand, etc.) is decidedly more favorable. Schorn et al. (1970) have suggested that, in low lying areas (surface pressure $>6.1 \mathrm{mb}$ ) when the terrain and temperature regime are favourable, there is a real possibility that liquid water exists - albeit only for short periods of time. This water might then be trapped for a substantial period of time - months perhaps - in the manner suggested by Sagan et al. In any case it should be perfectly clear that the possibility of liquid water on Mars cannot be dismissed out of hand on the basis of present evidence.

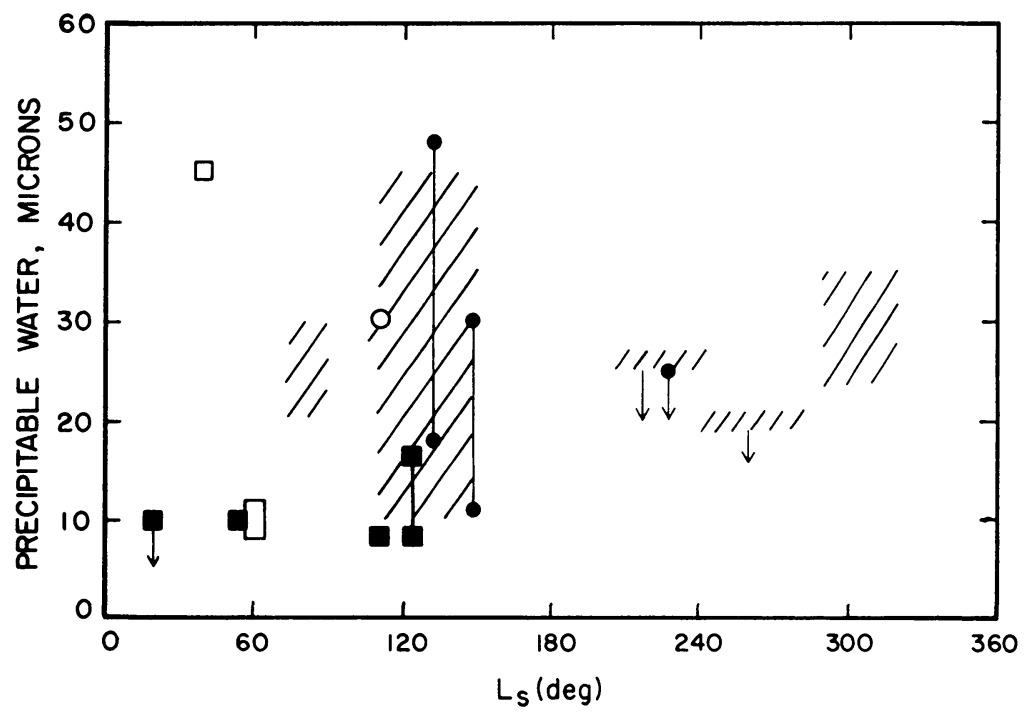

Fig. 2. Abundance determinations of Martian water vapor as a function of $L_{s}$. The open square represents Dollfus' 1963 observations; the open rectangle the 1963 results of Kaplan et al.; the filled squares the 1964-65 estimates of Schorn et al.; the open circle the 1969 observation of Owen and Mason; the filled circles the results of Tull in 1969. The individual results of a large number of McDonald spectra by Schorn, Farmer, Little, Barker, and Woszczyk are not plotted, but the approximate regions in which they fall are covered with diagonal lines. Vertical arrows point downward from observed upper limits and, in three cases, vertical lines connect the range of values found from a single plate. No attempt has been made to indicate probable or possible errors, which are substantial in all cases. All estimates have, however, been adjusted to utilize Farmer's improved line strengths for $225 \mathrm{~K}$. This illustration shows the general trend of $\mathrm{Martian} \mathrm{H}_{2} \mathrm{O}$ abundances with $L_{s}$.

Finally, the obvious questions arise - where did this water come from, and why this small, but definite amount? It does seem improbable that we are living precisely at that time when the last few cubic miles of water are escaping from Mars. Perhaps the $\mathrm{H}_{2} \mathrm{O}$ escape rate from Mars is much lower than we think. Perhaps water is added to the Martian atmosphere from the interior at just the right rate to make up for the escape rate. Perhaps a comet impact or igneous event has recently supplied the water. Perhaps. In my mind it does appear most likely to assume that in general, the origin of the water on Mars is similar to that of the origin of the water on Earth - it came 
from the body of the planet itself, whatever the time scales of production and escape may be. Such suggestions at present are, of course, just that. Mars, it appears, is still an engaging object for study and speculation.

\section{References}

Adamcik, J. A.: 1963, Planetary Space Sci. 11, 355.

Adams, W. S.: 1941, Astrophys. 93, 16.

Adams, W. S. and Dunham, T.: 1937, Publ. Astron. Soc. Pacific 49, 209.

Adams, W. S. and St. John, C.: 1926, Astrophys. J. 63, 133.

Barker, E. S., Schorn, R. A., Woszczyk, A., Tull, R. G., and Little, S. J.: 1970, Science, in press.

Benedict, W. S.: 1969, private communication to C. B. Farmer.

Campbell, W. W.: 1909, Lick Obs. Bull. 5, No. 150.

Campbell, W. W. and Albrecht, S.: 1910, Lick Obs. Bull. 6, No. 180, 11.

Delbouille, L. and Roland, G.: 1963, Mem. Soc. Roy. Sci. Liege, special Vol. 4, Liege.

de Vaucouleurs, Gerard: 1954, Physics of the Planet Mars, Faber and Faber, Ltd., London.

Dollfus, A.: 1948, Compt. Rend. Sci. 227, 331.

Dollfus, A.: 1951, Compt. Rend. Acad. Sci. 233, 467.

Dollfus, A.: 1961, in The Solar System, 3: Planets and Satellites (ed. by G. P. Kuiper and B. Middlehurst), University of Chicago Press, Chicago, p. 343.

Dollfus, A.: 1963, Compt. Rend. Acad. Sci. 256, 3009.

Dollfus, A.: 1964, Mem. Soc. Roy. Sci. Liege, Ve ser., IX, 392.

Dollfus, A.: 1965, Compt. Rend. Acad. Sci. 261, 1603.

Dunham, T.: 1952, in The Atmospheres of the Earth and Planets, 2nd ed., (ed. by G. P. Kuiper), University of Chicago Press, Chicago, p. 288.

Farmer, C. B.: Icarus, to be published.

Goody, R.: 1969, in Ann. Rev. Astron. Astrophys. 7 (ed. by L. Goldberg, D. Layzer, and J. G. Phillips), Annual Reviews, Inc., Palo Alto, Calif., 330.

Heyden, F. J., Kiess, C. C., and Willauer, W. R.: 1966, Astrophys. J. 143, 595.

Hess, S. L.: 1948, Publ. Astron. Soc. Pacific 60, 289.

Hess, S. L.: 1951, Rep. No. 9, Planetary Atmospheric Project, Lowell Obs., 172.

Hess, S. L.: 1961, in Advances on Space Science and Technology, Vol. 3, (ed. by F. I. Ordway), Academic Press, New York, p. 151.

Kaplan, L. D., Münch, G., and Spinrad, H.: 1964, Astrophys. J. 139, 1.

Kiess, C. C., Corliss, C. H., Kiess, H. K., and Corliss, E. L. R.: 1957, Astrophys. J. 116, 579.

Kiess, C. C., Corliss, C. H., and Kiess, H. K.: 1962, Astron. J. 67, 579.

Kiess, C. C., Karrer, S., and Kiess, H. K.: 1960, Publ. Astron. Soc. Pacific 72, 256.

Kiess, C. C., Karrer, S., and Kiess, H. K.: 1963, Publ. Astron. Soc. Pacific 75, 50.

Kuiper, G. P.: 1952, in The Atmospheres of the Earth and Planets (ed. by G. P. Kuiper), University of Chicago Press, Chicago, p. 306.

Lebedenskii, A. I. and Salova, G. I.: 1962, Soviet Astron.-AJ 6, 390.

Leighton, R. B. and Murray, B. C.: 1966, Science 153, 136.

Leovy, C.: 1966, Science 154, 1178.

Lowell, P. and Slipher, V. M.: 1905, Lowell Obs. Bull. No. 17, 116.

Lyot, B.: 1929, Ann. Obs. Meudon, VIII, 1, 51, 147.

Marshall, J. V.: 1964, Comm. Lunar Planetary Lab. 2, 167.

Moroz, V. I.: 1964, Soviet Astron.-AJ 8, 273.

Neugebauer, G., Münch, G., Chase, S. C., Hatzeubeler, H., Miner, E., and Schonfield, D.: 1969, Science 166, 98.

Owen, T. and Mason, H. P.: 1969, Science 165, 893.

Proctor, R. A.: 1873 ?, Other Worlds Than Ours, Hurst \& Company, New York.

Rank, D. H., Fink, U., Folz, J. V., and Wiggins, T. A.: 1964, Astrophys. J. 140, 366.

Russell, H. N., Dugan, R. S., and Stewart, J. Q.: 1945, Astronomy: I. The Solar System, rev. ed., Ginn and Company, Boston, p. 332.

Sagan, C.: 1961, Astron. J. 66, 52.

Sagan, C., Hanst, P. L., and Young, A. T.: 1965, Planetary Space Sci. 13, 73. 
Sagan, C., Levinthal, E. C., and Lederberg, J.: 1968, Science 159, 1191.

Schorn, R. A., Farmer, C. B., and Little, S. J.: 1970, Icarus, in press.

Schorn, R. A. J. and Young, L. D. G.: 1970, Icarus, to be published.

Schorn, R. A., Gray, L. D., and Barker, E. S.: 1969, Icarus 10, 241.

Schorn, R. A., Spinrad, H., Moore, R. C., Smith, H. J., and Giver, L. P.: 1947, Astrophys. J. 147, 743.

Sinton, W. M.: 1961, Publ. Astron. Soc. Pacific 73, 125.

Spinrad, H.: 1963, Publ. Astron. Soc. Pacific 75, 190.

Spinrad, H., Münch, G., and Kaplan, L.: 1963, Astrophys. J. 137, 1319.

Spinrad, H. and Richardson, E. H.: 1963, Icarus 2, 49.

Stoney, G. J.: 1898, Astrophys. J. 7, 25.

Tull, R. G.: 1970, Icarus, in press.

Urey, H. C.: 1959, in Handbuch der Physik (ed. by S. Flügge), Springer-Verlag, Berlin, p. 395.

Very, F. W.: 1909, Lowell Obs. Bull., Nos. 36 and 41.

Young, L. D. G.: 1969, Icarus 11, 386. 